\title{
A novel approach to cardiovascular disturbances in patients with schizophrenia spectrum disorders treated with long-acting injectable medication
}

This article was published in the following Dove Medical Press journal: Neuropsychiatric Disease and Treatment

\author{
Minodora Andor $1, *$ \\ Liana Dehelean ${ }^{2, *}$ \\ Ana-Maria Romosan ${ }^{2, *}$ \\ Valentina Buda ${ }^{3}$ \\ Gabriela Radu' \\ Florina Caruntu' \\ Aurora Bordejevic' \\ Minodora Marinela Manea ${ }^{4}$ \\ Ion Papava ${ }^{2}$ \\ Cristina Ana Bredicean ${ }^{2}$ \\ Radu Stefan Romosan ${ }^{2}$ \\ Mirela Tomescu' \\ 'Medical Semiology II, Department \\ of Internal Medicine I, "Victor \\ Babeș" University of Medicine \\ and Pharmacy, Timișoara, Romania; \\ ${ }^{2}$ Discipline of Psychiatry, Department \\ of Neurosciences, "Victor Babeș" \\ University of Medicine and Pharmacy, \\ Timisoara, Romania; ${ }^{3}$ Pharmacology \\ and Clinical Pharmacology, 2nd \\ Department, "Victor Babeș" \\ University of Medicine and Pharmacy, \\ Timișoara, Romania; ${ }^{4}$ Department of \\ Psychology, Medical Education, "Iuliu \\ Hațieganu" University of Medicine and \\ Pharmacy, Cluj-Napoca, Romania \\ *These authors contributed equally \\ to this work and thus share first \\ authorship
}

Correspondence: Liana Dehelean Discipline of Psychiatry, Department of Neurosciences, "Victor Babeș" University of Medicine and Pharmacy, 2 E. Murgu Square, Timisoara, Romania

Tel +40 723626328

Email lianadeh@umft.ro
Purpose: This research article assesses the cardiovascular impact of long-term injectable antipsychotic therapy on patients diagnosed with schizophrenia spectrum disorders. In our study, we attempted to quantify the potential causes of cardiovascular damage, assess cardiovascular parameters, and correlate them with the time elapsed from the onset of the psychosis until the initiation of injectable antipsychotic therapy, as well as the duration of long-acting therapy, and finally, to compare two of the most utilized long-acting injectable (LAI) medications (olanzapine vs risperidone). Patients and methods: This cross-sectional study recruited 64 patients of 2 outpatient clinics undergoing treatment with LAI antipsychotics for schizophrenic spectrum disorder. The study reports outpatients' clinical data, laboratory blood sample findings, routine echocardiography, as well as speckle tracking echocardiography.

Results: Among patients with longer durations of pre-long-acting antipsychotic treatment, body mass indices, mitral velocity wave values ( $\mathrm{E}$ and $\mathrm{A}$ waves), and the global longitudinal strain (GLS) measurements significantly correlated with patients' myocardial contractility. The study also found that GLS was significantly lower in the group in which pre-LAI duration was prolonged, and was not influenced by the duration of LAI treatment. Furthermore, patients receiving olanzapine showed significantly improved myocardial contractility as measured by the aforementioned parameters, in comparison with patients treated with risperidone.

Conclusion: The results of our study indicate that patients suffering from schizophrenia and who are left untreated or poorly treated for a longer period of time may develop myocardial impairment. The changes may be both secondary to a high prevalence of cardiovascular risk factors and may also be generated by the disease per se. The group who received olanzapine demonstrated improved results for a longer period of time without proper medication.

Keywords: schizophrenic patients, speckle tracking echocardiography, myocardial dysfunction

\section{Introduction}

With the introduction of long-acting injectable (LAI) formulations, antipsychotics may be administrated monthly or once every 3 months, promising more stable blood levels and remarkable results for the treatment of psychosis. ${ }^{11}$ Observational studies reflecting real-life conditions emphasize the superiority of the LAI medication in preventing psychotic recurrences. ${ }^{12}$ Nevertheless, for long-term treatments, it is important to thoroughly assess the side effects of medication. Antipsychotics may have an unfavorable impact on patients' mortality through their influence on metabolic and cardiovascular parameters. ${ }^{24,25}$

It is known that patients with psychosis have a lower life expectancy than the general population, ${ }^{15}$ most deaths being attributed to cardiovascular complications. ${ }^{6}$ Increased mortality and morbidity of cardiovascular disease in psychotic patients may be due 
to the underlying disease complications that cause anxiety and depression, but also to the accumulation of risk factors such as sedentary life, increased frequency of smoking and obesity, and a poor addressability to the cardiologist. ${ }^{10,16}$

Prospective $^{4}$ and retrospective ${ }^{6}$ studies have identified metabolic dysfunction in patients with psychosis, both before and after the introduction of antipsychotic medication. ${ }^{27}$ It seems that both the disease itself as well as the psychotropic medication would be involved in the cardiovascular pathology of these patients. ${ }^{23}$

In our study, we attempted to quantify the two potential causes of cardiovascular damage, by assessing patients' cardiovascular parameters such as blood pressure, electrocardiography, and echocardiography, and correlating them with the time elapsed from the onset of the psychosis to the beginning of the injectable antipsychotic therapy (pre-LAI) and the duration of the LAI therapy.

In order to develop a profile of cardiovascular risk for these patients, it is necessary to prioritize changes that may result in severe cardiovascular pathology. ${ }^{20}$ One must consider risk factors such as hyperlipidemia, smoking, confirmed high blood pressure, and an early diagnosis of myocardial damage. ${ }^{22}$

Left ventricular echocardiography, the classical approach to early myocardial damage detection, cannot routinely detect early contractility changes in these cases. ${ }^{21}$ Such changes occur only in the more advanced stages of myocardial contractility impairment. ${ }^{28}$

M-mode echocardiography may detect contractility changes but does not provide sufficient information regarding the number of affected segments, which can only be assessed by longitudinal and radial contractility determinations. While it is considered that ultrasound M-mode evaluation overestimates the systolic performance of the left ventricle (LV), strain rate imaging provides very accurate measurements of myocardial deformation. ${ }^{2,21}$ A large number of studies have demonstrated the utility of strain rates imaging in early detection of myocardial damage prior to LV ejection fraction (LVEF) depression. ${ }^{13}$ Numerous parameters exploring myocardial deformation, such as global longitudinal strain (GLS), global radial strain (GRS), and LV twist or torsion strain, ${ }^{26}$ are commonly used as non-invasive methods for the subclinical determination of myocardial damage.

GLS is a measurement used to evaluate left ventricular function in the longitudinal axis, allowing a closer study of subendocardial fibers, while GRS measurements are more closely linked to subepicardial fibers. ${ }^{29}$ An extensive evaluation of left ventricular contractility should consider both longitudinal and global strains.

\section{Patients and methods}

\section{Study population}

Sixty-four outpatients currently in remission (Brief Psychiatric Rating Scale - Expanded version 4.0 [BPRS-E] total score for the entire sample was $38.76 \pm 11.57)^{7}$ from two centers (Timisoara and Cluj-Napoca) and receiving treatment with LAI antipsychotics were analyzed in this cross-sectional study.

All the patients signed a written informed consent form to participate in the study. The inclusion criteria were: 1) diagnosis of schizophrenia or schizoaffective disorders according to ICD-10;2) all patients received LAIs olanzapine or risperidone (from the group of patients under medication, 2 patients were treated with olanzapine with 1 injection of $210 \mathrm{mg} / \mathrm{month}$ for $21 \pm 12.72$ months, 6 patients were treated with olanzapine with 1 injection of $300 \mathrm{mg} / \mathrm{month}$ for $17.83 \pm 14.23$ months, 6 patients were treated with olanzapine with 1 injection of $405 \mathrm{mg} /$ month for $13.9 \pm 11.87$ months, 18 patients were treated with olanzapine with 2 injections of $300 \mathrm{mg} / \mathrm{month}$ for $8.94 \pm 6.67$ months, 14 patients were treated with risperidone with 2 injections of $37.5 \mathrm{mg} / \mathrm{month}$ for $19.85 \pm 16.58$ months, and 18 patients were treated with risperidone with 2 injections of $50 \mathrm{mg} / \mathrm{month}$ for $30.0 \pm 21.5$ months); 3) age over 18 years; and 4) LVEF $>45 \%$. The exclusion criteria were: 1) previous diagnosis of ischemic heart disease or heart failure of any cause; 2) pregnancy or breastfeeding; and 3) previous use of any cardiovascular therapies. The study assessed the following parameters: cardiovascular risk factors (age, gender, smoking habits, and presence of the metabolic syndrome), transthoracic echocardiographic parameters, medication, and BPRS-E scores. The expanded version of BPRS was used to measure the severity of the psychiatric symptoms in patients with psychotic disorders. Fasting glycemia was measured using the PalmLab SC-101 glucometer. The lipid profile was determined using the LipidPro ILM-0001A lipid meter.

The study was carried out according to the Helsinki Declaration guidelines. The protocol of the study and the informed consent of the patients were approved by the Scientific Research Ethics Committee of "Victor Babes" University of Medicine and Pharmacy, Timisoara, Romania (Reference number 19/2015).

\section{Cardiological evaluation}

Cardiological evaluation was performed at the Cardiology Clinic of City Hospital, Timisoara. The echocardiography was performed by the same ultrasonographer using a VIVID S5 (GE Healthcare Bio-Sciences Corp., Piscataway, NY, USA) with a medium frequency transducer (3-8 MHz). 
M-mode, two-dimensional (2D), and Doppler echocardiography examinations were performed in accordance with the present guidelines. ${ }^{13}$ All images were digitally stored and analyzed offline with Echo Pac PC Dimension software (v 6.0). Doppler examination of the mitral valves was performed by measuring peak early (E) and late (A) filling velocities from the LV inflow pattern of the tips of mitral valves. The ejection fraction was calculated using Simpson's method. Three consecutive beats were used for measurement of each parameter.

Cardiac rotation was assessed using 2D strain imaging. GLS was determined using three apical planes: two-chamber, three-chamber, and four-chamber views. Segments that failed to track were excluded. GRS was calculated using peak systolic radial strain values in all six segments of the perfectly rounded parasternal short-axis view of the mitral valve of the papillary muscle and apical level.

Patients were treated with injectable antipsychotic medication in an outpatient setting to avoid non-compliance. The antipsychotic medication was administered randomly.

In order to discriminate between the pharmacologicaland disorder-induced effects, we assessed the following time intervals: the duration of the psychosis, defined as the number of months between the onset of the disease and present assessment of the patient; the duration of LAI treatment; and finally, the time from patient's onset of the psychiatric condition to the onset of LAI treatment (pre-LAI period). ${ }^{7}$

\section{Statistical analysis}

Statistical analysis was performed using IBM SPSS Statistic (version 20). Comparison between different series of values was performed by Mann-Whitney $U$-test due to the non-Gaussian distribution of the measured parameters, as evidenced by the Shapiro-Wilk test. A chi-squared test was used for categorical variables. Correlation intensity was expressed by the Spearman correlation coefficient.

\section{Results}

\section{Demographic and clinical data of the patients}

Of the 64 patients enrolled in the study, $57.8 \%$ were men, and the mean age of the group at study onset was $41.25 \pm 11.14$ years. The mean age of the disorder onset was $29.54 \pm 10.45$ years (28.45 \pm 10.66 in men and $31.03 \pm 10.15$ in women). The diagnosis of schizophrenia was established for 51 patients (28 men, 23 women) and schizoaffective disorder for 13 patients ( 9 men, 4 women).

Of the recruited groups, $40.6 \%$ were smokers, with a mean use of 8.98 cigarettes/day.

\section{Assessing the cardiovascular risk factors of the studied group according to the duration of the disease or the injectable treatment}

An analysis was performed to verify whether disease duration before treatment initiation (pre-LAI) and the duration of the treatment (LAI) had any influence upon metabolic syndrome parameters (SBP, DPB, hyperglycemia, hypercholesterolemia, hypertriglyceridemia, obesity, and smoking). The approach used in this analysis is somewhat similar to that of retrospective studies: for each cardiovascular risk factor, the sampled population was divided into two groups: one for which the risk factor was present (marked with "+" in the table) and the other for which the risk factor was absent ("-"), using the usual cut-point values for this split. Then we compared their corresponding pre-LAI (or LAI or total) durations using the Mann-Whitney test (for distributions different from normal). Thus, a significant difference might be interpreted as a significant presence of that factor in a particular patient group; in our study, the group included those patients with a longer pre-LAI (or LAI or total) duration. The results of the analysis are presented in Table 1.

Analysis of patients' blood pressure data and blood sample parameters, such as glycemia, total cholesterol, and triglyceridemia, found no statistically significant differences among the patients. The only parameter from this category which was significantly increased in the patients with a longer duration of the LAI treatment was the body mass index.

\section{Echocardiographic parameters in the studied population}

The same statistical approach was also used to highlight the possible impact of long-term treatment on echocardiographic parameters. For each of the measured parameters, the whole sample was divided into two groups, using the usual cut-point values; then, we compared their corresponding pre-LAI, LAI, and total durations using the Mann-Whitney $U$-test. The results are presented in Table 2 , where $n=$ number of patients in each group. The table presents the median durations for each group and the test results: $U$ and $P$-value (asymptotic two-tailed sigma), respectively.

Our analysis of the sampled population found no differences regarding left atrium dimensions before or after medication, with most patients presenting abnormal left atrium volume index.

Reduced values of the $\mathrm{E}$ and $\mathrm{A}$ waves were found more often $(P<0.05)$ among patients with long pre-LAI treatment durations. Similarly, elevated values for the E/A ratio and 
Table I Median duration of disease and duration of LAl treatment (months) depending on components of metabolic syndrome cardiovascular risk factors

\begin{tabular}{|c|c|c|c|c|c|c|}
\hline $\begin{array}{l}\text { Cardiovascular risk factors and } \\
\text { conditions with cut-point values }\end{array}$ & Groups and tests & $\begin{array}{l}\text { Computed } \\
\text { parameters }\end{array}$ & $\mathbf{n}$ & Pre-LAI & LAI & Total duration \\
\hline \multirow[t]{4}{*}{ Systolic hypertension (>I40 mmHg) } & \multirow[t]{2}{*}{ Groups } & + & 7 & 120 & 12 & 156 \\
\hline & & - & 57 & 69 & 12 & 84 \\
\hline & \multirow{2}{*}{$\begin{array}{l}\text { Mann-Whitney } \\
\text { test }\end{array}$} & $U$ & & 136 & 134 & 136.5 \\
\hline & & $P$-value (two-tailed) & & 0.172 & 0.166 & 0.175 \\
\hline \multirow[t]{4}{*}{ Diastolic hypertension $(>90 \mathrm{mmHg})$} & \multirow[t]{2}{*}{ Groups } & + & 2 & 228 & 42 & 270 \\
\hline & & - & 62 & 74.5 & 12 & 84 \\
\hline & \multirow{2}{*}{$\begin{array}{l}\text { Mann-Whitney } \\
\text { test }\end{array}$} & $U$ & & 25 & 30.5 & 21 \\
\hline & & $P$-value (two-tailed) & & 0.153 & 0.222 & 0.113 \\
\hline \multirow[t]{4}{*}{ Hyperglycemia (>106 mg/dL) } & \multirow[t]{2}{*}{ Groups } & + & 41 & 81 & 12 & 84 \\
\hline & & - & 23 & 77 & 11 & 96 \\
\hline & \multirow{2}{*}{$\begin{array}{l}\text { Mann-Whitney } \\
\text { test }\end{array}$} & $U$ & & 460 & 421 & 458 \\
\hline & & $P$-value (two-tailed) & & 0.872 & 0.483 & 0.850 \\
\hline \multirow[t]{4}{*}{ Hypercholesterolemia ( $>200 \mathrm{mg} / \mathrm{dL})$} & \multirow[t]{2}{*}{ Groups } & + & 46 & 74.5 & 8 & 120 \\
\hline & & - & 18 & 90 & 12 & 84 \\
\hline & \multirow{2}{*}{$\begin{array}{l}\text { Mann-Whitney } \\
\text { test }\end{array}$} & $U$ & & 408.5 & 354.5 & 410 \\
\hline & & $P$-value (two-tailed) & & 0.935 & 0.373 & 0.958 \\
\hline \multirow[t]{4}{*}{ Hypertriglyceridemia (>150 mg/dL) } & \multirow[t]{2}{*}{ Groups } & + & 32 & 84 & 12.5 & 84 \\
\hline & & - & 32 & 70.5 & 11.5 & 96 \\
\hline & \multirow{2}{*}{$\begin{array}{l}\text { Mann-Whitney } \\
\text { test }\end{array}$} & $U$ & & 447 & 420.5 & 476.5 \\
\hline & & $P$-value (two-tailed) & & 0.383 & 0.218 & 0.633 \\
\hline \multirow[t]{4}{*}{ Obesity (BMI >30 kg/m²) } & \multirow[t]{2}{*}{ Groups } & + & 22 & 114 & 24 & 156 \\
\hline & & - & 42 & 69 & 10 & 72 \\
\hline & \multirow{2}{*}{$\begin{array}{l}\text { Mann-Whitney } \\
\text { test }\end{array}$} & $U$ & & 403 & 239.5 & 372.5 \\
\hline & & $P$-value (two-tailed) & & 0.404 & 0.002 & 0.205 \\
\hline
\end{tabular}

Notes: The "+" groups include the patients for whom the condition is true, the rest are included in the "-" group. The Mann-Whitney test was applied between the "+" and the "-" groups. P-values for significant differences are presented in bold.

Abbreviations: BMI, body mass index; LAI, long-acting injectable.

isovolumic relaxation time (IVRT) were statistically more frequent among long pre-LAI treatment patients. However, when considering LAI durations, the same parameters did not produce significant differences.

It is known that mitral $\mathrm{E}$ velocity is affected by alterations in the rate of LV relaxation, and mitral A wave velocity is affected by LV compliance and left atrial contractile function.

The duration of IVRT, a marker of poor myocardial relaxation, was found to be significantly prolonged among patients whose treatment initiation had been delayed.

While LVEF values did not statistically differ between the two groups, it was found that GLS values were significantly lower among patients with longer pre-LAI durations. Furthermore, GLS values were not influenced by the duration of LAI treatments.

LV longitudinal systolic function, detected by LV GLS via speckle tracking, can be used as an indicator of myocardial dysfunction. Lower values of GLS indicate impaired LV global longitudinal function.
The final type of analysis presented here refers to a comparison between the two treatments - olanzapine vs risperidone. The Mann-Whitney $U$-test shows that patients treated with olanzapine presented significantly higher values of the following parameters, with respect to patients treated risperidone (Table 3):

- E wave ( $U=344, Z=-2.251, P=0.024)$

- $\operatorname{LV}$ GLS ( $U=351, Z=-2,157, P=0.031)$

- $\operatorname{LV}$ GRS $(U=310.5, Z=-2.701, P=0.007)$.

\section{Discussion}

According to our results, a delay in initiating the LAI treatment of patients with schizophrenia spectrum disorders is a statistically relevant risk factor for cardiovascular impairment, the relaxation dysfunction of the LV, and also an impaired GLS. The early stages of affected myocardial relaxation, detected echocardiographically through changes in E and A velocities, E/A ratio, prolonged IVRT, 
Table 2 Median duration of disease and duration of treatment with LAI (months) according to echocardiography parameters

\begin{tabular}{|c|c|c|c|c|c|c|}
\hline $\begin{array}{l}\text { Echocardiographic parameters and } \\
\text { conditions with cut-point values }\end{array}$ & Groups and tests & $\begin{array}{l}\text { Computed } \\
\text { parameters }\end{array}$ & $\mathbf{n}$ & Pre-LAI & LAI & Total duration \\
\hline \multirow[t]{4}{*}{ LAVI $\left(m L / m^{2}\right)$} & \multirow[t]{2}{*}{ Groups } & + & 8 & 87.5 & 16 & 92 \\
\hline & & - & 56 & 71.3 & 11 & 87 \\
\hline & \multirow[t]{2}{*}{ Mann-Whitney test } & $U$ & & 306 & 258.5 & 352 \\
\hline & & $P$-value (two-tailed) & & 0.262 & 0.095 & 0.412 \\
\hline \multirow[t]{4}{*}{ IVS >II mm } & \multirow[t]{2}{*}{ Groups } & + & 9 & 108 & 10 & 132 \\
\hline & & - & 55 & 77 & 12 & 84 \\
\hline & \multirow[t]{2}{*}{ Mann-Whitney test } & $U$ & & 192 & 220 & 198 \\
\hline & & $P$-value (two-tailed) & & 0.284 & 0.594 & 0.338 \\
\hline \multirow[t]{4}{*}{ PWLV > II mm } & \multirow[t]{2}{*}{ Groups } & + & 8 & 132 & 12 & 144 \\
\hline & & - & 56 & 74.5 & 12 & 84 \\
\hline & \multirow[t]{2}{*}{ Mann-Whitney test } & $U$ & & 182.5 & 209 & 182.5 \\
\hline & & $P$-value (two-tailed) & & 0.399 & 0.760 & 0.399 \\
\hline \multirow[t]{4}{*}{$\mathrm{EDDLV}>56 \mathrm{~mm}$} & \multirow[t]{2}{*}{ Groups } & + & 3 & 120 & 24 & 156 \\
\hline & & - & 61 & 77 & 12 & 84 \\
\hline & \multirow[t]{2}{*}{ Mann-Whitney test } & $U$ & & 73.5 & 87 & 77 \\
\hline & & $P$-value (two-tailed) & & 0.587 & 0.886 & 0.645 \\
\hline \multirow[t]{4}{*}{ E wave $<80 \mathrm{~m} / \mathrm{s}$} & \multirow[t]{2}{*}{ Groups } & + & 49 & 96 & 12 & 108 \\
\hline & & - & 15 & 24 & 12 & 48 \\
\hline & \multirow[t]{2}{*}{ Mann-Whitney test } & $U$ & & 194 & 357 & 197 \\
\hline & & $P$-value (two-tailed) & & 0.006 & 0.867 & 0.007 \\
\hline \multirow[t]{4}{*}{ A wave $<60 \mathrm{~m} / \mathrm{s}$} & \multirow[t]{2}{*}{ Groups } & + & 35 & 45 & 10 & 60 \\
\hline & & - & 29 & 108 & 14 & 156 \\
\hline & \multirow[t]{2}{*}{ Mann-Whitney test } & $U$ & & 335.5 & 384 & 335.5 \\
\hline & & $P$-value (two-tailed) & & 0.020 & 0.096 & 0.020 \\
\hline \multirow[t]{4}{*}{$E / A>0.8$} & \multirow[t]{2}{*}{ Groups } & + & 48 & 92 & 15 & 107 \\
\hline & & - & 16 & 26 & 17 & 53 \\
\hline & \multirow[t]{2}{*}{ Mann-Whitney test } & $U$ & & 189 & 442 & 180 \\
\hline & & $P$-value (two-tailed) & & 0.012 & 0.052 & 0.023 \\
\hline \multirow[t]{4}{*}{ IVRT $>100 \mathrm{~ms}$} & \multirow[t]{2}{*}{ Groups } & + & 46 & 82 & 19 & 81 \\
\hline & & - & 18 & 44 & 23 & 67 \\
\hline & Mann-Whitney test & $U$ & & 155 & 224 & 132 \\
\hline & & $P$-value (two-tailed) & & 0.044 & 0.058 & 0.053 \\
\hline LVEF $<50 \%$ & Groups & + & 8 & 207 & 24 & 258 \\
\hline & & - & 56 & 70.5 & 12 & 84 \\
\hline & Mann-Whitney test & $U$ & & 156 & 159 & 154.5 \\
\hline & & $P$-value (two-tailed) & & 0.157 & 0.185 & 0.158 \\
\hline GLS $<21.5 \%$ & Groups & + & 44 & 109.5 & 12 & 120 \\
\hline & & - & 20 & 28.5 & 11.5 & 60 \\
\hline & Mann-Whitney test & $U$ & & 241 & 431 & 260.5 \\
\hline & & $P$-value (two-tailed) & & 0.004 & 0.896 & 0.009 \\
\hline GRS $<45 \%$ & Groups & + & 48 & 82.5 & 12 & 90 \\
\hline & & - & 16 & 74.5 & 10 & 90 \\
\hline & Mann-Whitney test & $U$ & & 346.5 & 288 & 342 \\
\hline & & $P$-value (two-tailed) & & $0.56 \mathrm{I}$ & 0.135 & 0.514 \\
\hline
\end{tabular}

Notes: The "+" groups include the patients for whom the condition is true, the rest are included in the "-" group. The Mann-Whitney test was applied between the "+" and the "-" groups. P-values for significant differences are presented in bold.

Abbreviations: EDDLV, end diastolic diameter of the left ventricle; GLS, global longitudinal strain; GRS, global radial strain; IVS, interventricular septum; IVRT, isovolumetric relaxation time; LAI, long-acting injectable; LAVI, left atrium volume index; LVEF, left ventricle ejection fraction; PWLV, posterior wall of the left ventricle. 
Table 3 Comparison of echocardiographic parameters between olanzapine and risperidone (Mann-Whitney $U$ test results)

\begin{tabular}{|l|l|l|l|l|l|l|l|l|l|l|}
\hline Statistical parameters & LAVI & RV & IVS & PWLV & EDDLV & LVEF & E wave & A wave & GLS & GRS \\
\hline Mann-Whitney U & 434.0 & 509.5 & 503.5 & 495.0 & 493.0 & 435.5 & 344.0 & 421.0 & 351.0 & 310.5 \\
Wilcoxon W & 930.0 & $1,070.5$ & $1,064.5$ & $1,056.0$ & $1,054.0$ & 931.5 & 840.0 & 982.0 & 847.0 & 806.5 \\
Z & -1.045 & -0.027 & $-0.11 \mathrm{I}$ & -0.226 & -0.250 & -1.024 & -2.251 & -1.217 & -2.157 & -2.701 \\
Asymp Sig (two-tailed) & 0.296 & 0.978 & 0.912 & 0.821 & 0.803 & 0.306 & $\mathbf{0 . 0 2 4}$ & 0.223 & $\mathbf{0 . 0 3 I}$ & $\mathbf{0 . 0 0 7}$ \\
\hline
\end{tabular}

Note: $P$-values for significant differences are presented in bold.

Abbreviations: EDDLV, end diastolic diameter of the left ventricle; GLS, global longitudinal strain; GRS, global radial strain; IVS, interventricular septum; LAVI, left atrium volume index; LVEF, left ventricle ejection fraction; PWLV, posterior wall of the left ventricle; RV, right ventricle.

and decreased GLS, are correlated with the duration of the pre-LAI period.

It is known that patients with schizophrenia have a worse cardiovascular risk profile than the general population. ${ }^{1,3-5}$ According to our results, although the data showed hypertension, hyperglycemia, and hypercholesterolemia, there were no significant changes among these parameters between the duration of pre-LAI treatment periods and LAI treatment periods; therefore, LV impairment may not be entirely attributable to coronary atherosclerotic disease in these patients.

At the end of LAI treatment, patients' LV relaxation showed improvement, so we may infer that proper pharmaceutical treatment can be shown to lower the risk of diminished myocardial contractility.

In our study, the $2 \mathrm{D}$ strain method proved to be a more useful tool for the early detection of subclinical myocardial impairment. GLS was more markedly reduced in the patients who suffered from the disease for a longer period of time before initiating LAI treatment. It is known that GLS is useful in detecting myocardial damage before any changes in LVEF. ${ }^{28}$

According to our results, treatment with both olanzapine and risperidone improved myocardial contractility, as assessed by GLS and GRS. Among the groups divided according to the LAI medication received, the group on olanzapine had better results on echocardiographic parameters which show the LV contractility. ${ }^{17}$

An enlarged left atrium is strongly suggestive of elevated LV filling pressure, when excluding comorbidities which can cause the enlargement of the left atrium, but a normal left atrium volume is often seen in the earliest stages of diastolic dysfunction.

The mechanisms probably involved in the depressed myocardial contractility during pre-LAI are coronary spasm and sympathetic-mediated cardiac arrhythmias. ${ }^{30}$ Serotonin (5-HT) activity is linked to depression, anxiety, and myocardial impairment secondary to coronary disease. ${ }^{8}$

Several cases of LAI-related myocarditis or cardiomyopathy are also described in medical literature, ${ }^{9,14}$ but most cases refer to patients treated with clozapine. ${ }^{18}$ Eosinophilic myocarditis was the favored etiology and was considered to be a direct toxicity or allergic reaction. ${ }^{19}$
The strengths of the present study consist of the patients' constant adherence to medication and dosage, as well as the extensive cardiovascular assessment, including both routine echocardiography and speckle tracking echocardiography, in order to assess the early cardiac changes.

\section{Limitations}

The limitations of the study are the relatively low number of assessed patients, especially when the groups are divided according to different treatments and the duration of the disease before treatment, which is far longer than the duration on the treatment; this may be a minor shortcoming with respect to the statistical results.

\section{Conclusion}

Two-dimensional strain analysis allows detection of early, subtle myocardial alterations, and is able to unmask the small, subclinical changes of myocardial deformation with greater accuracy than that of classical echocardiography.

The results from our study indicate that patients suffering from schizophrenia who go untreated or are poorly treated for longer periods of time may develop myocardial impairment. The changes are likely related to the high prevalence of metabolic syndrome, but are also generated by the disease per se.

This may help target patients with other comorbidities known to cause irreversible cardiovascular damage, enabling them to be closely monitored to ensure they would benefit from early initiation of cardiovascular medical therapy.

\section{Author contributions}

MA, LD, AMR: study concept and design, study supervision, critical revision of manuscript for intellectual content, and statistical analysis; VB, GR, FC, AB: acquisition of data, analysis and interpretation of data, statistical analysis, writing of article; MMM, IP, CAB, RSR: acquisition of data, study supervision, and critical revision of manuscript for intellectual content; and MT: study supervision. All authors contributed toward data analysis, drafting and critically revising the paper, gave final approval of the version to be published, and agreed to be accountable for all aspects of the work. 


\section{Disclosure}

The authors report no conflicts of interest in this work.

\section{References}

1. Alberti KG, Zimmet P, Shaw J. Metabolic syndrome - a new world-wide definition. A consensus statement from the international diabetes federation. Diabet Med. 2006;23(5):469-480.

2. Aurigemma GP, Silver KH, Priest MA, Gaasch WH. Geometric changes allow normal ejection fraction despite depressed myocardial shortening in hypertensive left ventricular hypertrophy. J Am Coll Cardiol. 1995; 26(1):195-202.

3. Carrillo JA, Herráiz AG, Ramos SI, Gervasini G, Vizcaíno S, Benítez J. Role of the smoking-induced cytochrome P450 (CYP)1A2 and polymorphic CYP2D6 in steady-state concentration of olanzapine. J Clin Psychopharmacol. 2003;23(2):119-127.

4. Citrome L, Collins JM, Nordstrom BL, et al. Incidence of cardiovascular outcomes and diabetes mellitus among users of second-generation antipsychotics. J Clin Psychiatry. 2013;74(12):1199-1206.

5. Coulter DM, Bate A, Meyboom RH, Lindquist M, Edwards IR. Antipsychotic drugs and heart muscle disorder in international pharmacovigilance: data mining study. BMJ. 2001;322(7296):1207-1209.

6. Davidson S, Judd F, Jolley D, Hocking B, Thompson S, Hyland B. Cardiovascular risk factors for people with mental illness. Aust $N Z J$ Psychiatry. 2001;35(2):196-202.

7. Dehelean L, Andor M, Romoşan AM, Tomescu MC. Pharmacological and disorder associated cardiovascular changes in patients with psychosis. A comparison between olanzapine and risperidone. Farmacia. 2018;66(1):129-134.

8. Emul M, Kalelioglu T. Etiology of cardiovascular disease in patients with schizophrenia: current perspectives. Neuropsychiatr Dis Treat. 2015;11:2493-2503.

9. Montastruc G, Favreliere S, Sommet A, et al. Drugs and dilated cardiomyopathies: a case/noncase study in the French PharmacoVigilance Database. Br J Clin Pharmacol. 2010;69(3):287-294.

10. Heiskanen T, Niskanen L, Lyytikäinen R, Saarinen PI, Hintikka J. Metabolic syndrome in patients with schizophrenia. J Clin Psychiatry. 2003;64(5):575-579.

11. Kim DH, Maneen MJ, Stahl SM. Building a better antipsychotic: receptor targets for the treatment of multiple symptom dimensions of schizophrenia. Neurotherapeutics. 2009;6(1):78-85.

12. Kishimoto T, Nitta M, Borenstein M, Kane JM, Correll CU. Long-acting injectable versus oral antipsychotics in schizophrenia: a systematic review and meta-analysis of mirror-image studies. J Clin Psychiatry. 2013;74(10):957-965.

13. Lang RM, Badano LP, Mor-Avi V, et al. Recommendations for cardiac chamber quantification by echocardiography in adults: an update from the American Society of Echocardiography and the European Association of Cardiovascular Imaging. Eur Heart J Cardiovasc Imaging. 2015; 16(3):233-271.

14. Lee TW, Tsai SJ, Hwang JP. Severe cardiovascular side effects of olanzapine in an elderly patient: case report. Int J Psychiatry Med. 2003;33(4):399-401.
15. Lehman AF, Steinwachs DM. Translating research into practice: the Schizophrenia Patient Outcomes Research Team (PORT) treatment recommendations. Schizophr Bull. 1998;24(1):1-10.

16. Mcevoy JP, Meyer JM, Goff DC, et al. Prevalence of the metabolic syndrome in patients with schizophrenia: baseline results from the Clinical Antipsychotic Trials of Intervention Effectiveness (CATIE) schizophrenia trial and comparison with national estimates from NHANES III. Schizophr Res. 2005;80(1):19-32.

17. Megens AA, Awouters FH, Schotte A, et al. Survey on the pharmacodynamics of the new antipsychotic risperidone. Psychopharmacology (Berl). 1994;114(1):9-23.

18. Murch S, Tran N, Liew D, Petrakis M, Prior D, Castle D. Echocardiographic monitoring for clozapine cardiac toxicity: lessons from real-world experience. Australas Psychiatry. 2013;21(3):258-261.

19. Nasrallah HA. Atypical antipsychotic-induced metabolic side effects: insights from receptor-binding profiles. Molecular Psychiatry. 2008;13(1): 27-35.

20. O'Brien P, Oyebode F, O'Brien P, Femi O. Psychotropic medication and the heart. Adv Psychiatric Treat. 2003;9(6):414-423.

21. Paulus WJ, Tschöpe C, Sanderson JE, et al. How to diagnose diastolic heart failure: a consensus statement on the diagnosis of heart failure with normal left ventricular ejection fraction by the Heart Failure and Echocardiography Associations of the European Society of Cardiology. Eur Heart J. 2007;28(20):2539-2550.

22. Remington G. Schizophrenia, antipsychotics, and the metabolic syndrome: is there a silver lining? Am J Psychiatry. 2006;163(7):1132-1134.

23. Ringen PA, Engh JA, Birkenaes AB, Dieset I, Andreassen OA. Increased mortality in schizophrenia due to cardiovascular disease - a non-systematic review of epidemiology, possible causes, and interventions. Front Psychiatry. 2014;5:137.

24. Robinson DS. Insulin secretion and psychotropic drugs. Prim Psych. 2006;13:26-27.

25. Saari KM, Lindeman SM, Viilo KM, et al. A 4-fold risk of metabolic syndrome in patients with schizophrenia: the Northern Finland 1966 Birth Cohort study. J Clin Psychiatry. 2005;66(5):559-563.

26. Sengeløv M, Jørgensen PG, Jensen JS, et al. Global longitudinal strain is a superior predictor of all-cause mortality in heart failure with reduced ejection fraction. JACC Cardiovasc Imaging. 2015;8(12):1351-1359.

27. Simon V, van Winkel R, de Hert M. Atypical antipsychotics dose dependent? A literature review. J Clin Psych. 2009;70(7):1041-1050.

28. Sutherland GR, Di Salvo G, Claus P, D’Hooge J, Bijnens B. Strain and strain rate imaging: a new clinical approach to quantifying regional myocardial function. J Am Soc Echocardiogr. 2004;17(7):788-802.

29. Vinereanu D, Lim PO, Frenneaux MP, Fraser AG. Reduced myocardial velocities of left ventricular long-axis contraction identify both systolic and diastolic heart failure-a comparison with brain natriuretic peptide. Eur J Heart Fail. 2005;7(4):512-519.

30. Wilson PWF, D’Agostino RB, Levy D, Belanger AM, Silbershatz H, Kannel WB. Prediction of coronary heart disease using risk factor categories. Circulation. 1998;97(18):1837-1847.
Neuropsychiatric Disease and Treatment

\section{Publish your work in this journal}

Neuropsychiatric Disease and Treatment is an international, peerreviewed journal of clinical therapeutics and pharmacology focusing on concise rapid reporting of clinical or pre-clinical studies on a range of neuropsychiatric and neurological disorders. This journa is indexed on PubMed Central, the 'PsycINFO' database and CAS,

\section{Dovepress}

and is the official journal of The International Neuropsychiatric Association (INA). The manuscript management system is completely online and includes a very quick and fair peer-review system, which is all easy to use. Visit http://www.dovepress.com/testimonials.php to read real quotes from published authors. 